\title{
Medidas de atividade física em crianças e adolescentes com deficiência: uma revisão sistemática
}

\section{Giovanna Carla Interdonato ${ }^{1,3}$ Márcia Greguol ${ }^{2,3}$}

1. Programa de Pós- Graduação em Educação Física da Universidade Estadual de Londrina (UEL), Londrina, PR, Brasil. 2. Departamento de Esporte da Universidade Estadual de Londrina (UEL), Londrina, PR, Brasil.

3. Grupo de Estudo e Pesquisa em Atividade Física e Deficiência - GEPAFID, Londrina, PR, Brasil.

\section{ENDEREÇOPARA CORRESPONDÊNCIA}

Giovanna Carla Interdonato Rua Borba Gato, 1158 - ap. 601 Vila Ipiranga - Londrina - PR 86010630

giointerdonato@hotmail.com

$\begin{array}{ll}\text { - Recebido: } & 11 / 12 / 2010 \\ \text { - Re-submissão: } & 16 / 03 / 2011 \\ & 16 / 05 / 2011 \\ & 18 / 08 / 2011 \\ \text { - Aceito: } & 08 / 09 / 2011\end{array}$

\section{Resumo}

O objetivo do estudo foi analisar os principais métodos de mensuração de atividade física utilizados em crianças e adolescentes com deficiência. Foi realizada uma revisão sistemática da literatura através das bases de dados: SportDiscus, Medline, Lilacs e Scielo. Foram selecionados estudos que analisaram a prática habitual de atividade física em crianças e adolescentes com deficiência, publicados entre os anos de 2000 e 2010. Também foi verificado o tipo de método de mensuração utilizado e os resultados obtidos. No total, 11 estudos foram incluídos. A forma de mensuração da atividade física mais utilizada foi a acelerometria ( $\mathrm{n}=$ 6). A duração da utilização dos diferentes instrumentos de mensuração foi de 1 dia a duas semanas, variando de acordo com o tipo de método utilizado, o tipo de deficiência da amostra e o objetivo do estudo. Todavia, não existiu um consenso quanto ao tipo de método mais correto a ser utilizado para mensurar os níveis de atividade física em jovens com deficiência. A acelerometria foi o método de mensuração mais utilizado pelos autores nos estudos encontrados. Observou-se ainda que o tipo de método a ser utilizado tem como critério de escolha comum principalmente o tipo de deficiência apresentada pela amostra e o objetivo do estudo, a fim de que se torne viável a realização da pesquisa .

Palavras-chave: Atividade física; Crianças com deficiência; Saúde.

\section{Abstract}

The purpose of the study was to analyze the main methods of physical activity measurement used in children and adolescents with disabilities. It was performed a systematic literature review through the databases: SportDiscus, Medline, Lilacs and Scielo. Were selected studies that examined physical activity practice in children and adolescents with disabilities, published between 2000 and 2010. It was also verified the type of measurement method used and results obtained. In total, 11 studies were included. The most used method of physical activity measurement was accelerometry $(n=6)$. Duration of use of different measurement instruments was one day to two weeks, varying with the type of method used, type of disability of the sample and objective of the study. However, there wasn't a consensus about the most correct method to be used to measure physical activity levels in young people with disabilities. According to the studies, accelerometry was the most used method of measurement. It was also observed that the method to be used is chosen according to the type of disability presented by the sample and the objective of the study, so that research may become feasible.

Keywords: Physical activity; Disabled children; Health. 


\section{INTRODUÇÃO}

Os benefícios promovidos pela prática habitual de atividade física são muito evidenciados na literatura nos dias atuais. Alguns autores apontam que o desenvolvimento de hábitos, comportamentos e atitudes descritoras de um estilo de vida saudável e ativo nos indivíduos tende a ocorrer ainda na infância, prosseguindo na fase escolar e podendo acompanhá-lo durante toda a fase adulta ${ }^{1,2}$. Dessa forma, têm sido foco de atenção dos profissionais da saúde estudos voltados à mensuração dos níveis de atividade física entre crianças e adolescentes $^{3}$.

No entanto, apesar de se saber da importância dos benefícios que a atividade física promove, nota-se na literatura que ainda são poucos os estudos voltados à atividade física para pessoas que possuem algum tipo de deficiência. Além disso, os estudos encontrados apresentam procedimentos metodológicos variados, bem como amostras com diferentes tipos de deficiência, o que torna difícil a comparação dos dados obtidos.

De acordo com Interdonato e Greguol ${ }^{4}$, a prática de atividades físicas voltada às pessoas com algum tipo de deficiência poderá contribuir para a manutenção de sua saúde e capacidades funcionais, aumentando ainda suas habilidades motoras e desenvolvendo a autoestima e autoconfiança, características estas que são de extrema importância na adolescência, fase de constantes mudanças no estado de humor e de queda do nível de atividade física habitual.

Todavia, quando se procura um indicador para a prática habitual de atividade física, diversas questões têm sido levantadas, desde a definição até a utilização de instrumentos de medida para pessoas com deficiência. Assim, algumas pesquisas recentes têm destacado a importância do tema, propondo inclusive a criação de instrumentos específicos para a utilização com estes indivíduos ${ }^{5}$.

Segundo Cervantes e Porretta ${ }^{6}$, ainda existem muitas perguntas sem resposta quando se trata da questão da prática de atividade física por pessoas com deficiência, como por exemplo o quanto de atividade efetivamente é realizada por esta população ou ainda qual a quantidade ideal a ser realizada tendo em vista a promoção da saúde. Ainda de acordo com os autores, são necessários estudos focados na disseminação dos métodos de mensuração da atividade física de pessoas com deficiência, quando possível evidenciando os aspectos relacionados à validade e reprodutibilidade dos mesmos.

Em pessoas com algum tipo de deficiência, assim como em outras populações pesquisadas, verifica-se a escassez de estudos representativos e de base populacional sobre o tema relacionado aos níveis de atividade física. Além disso, os resultados encontrados não são convergentes muitas vezes com a realidade existente, variando bastante na forma de mensuração e no tipo de instrumento utilizado. Com o intuito de expor tal realidade existente, o objetivo do presente estudo foi analisar, por meio de uma revisão sistemática da literatura, os principais métodos de mensuração de atividade física utilizados em crianças e adolescentes com deficiência.

\section{MÉTODOS}

A revisão foi iniciada pela busca de estudos bibliográficos que procuraram analisar a prática habitual de atividade física em crianças e adolescentes com deficiência. Focou-se em estudos realizados dentro do período de 2000 a 2010, sobretudo pela maior concentração de publicação pertinentes ao tema neste período. Inicialmente foi realizada uma busca integrada no portal de periódicos da CAPES, onde se verificou em quais bases de dados encontravam-se artigos na temática pesquisada.

Em seguida, foram consultadas as bases de dados que possuíam os artigos, sendo elas: SportDiscus, Medline, Lilacs e Scielo. Foram utilizados os seguintes termos em português e inglês, com base no problema investigado no estudo: atividade física/ physical activity, deficiência / disability e impairment, crianças / children, adolescentes / youngs, mensuração / meansurements, pedometria / pedometers, acelerometria / acelerometers. Estes descritores foram colocados na busca isoladamente e posteriormente combinados em grupos de dois, três e quatro termos simultaneamente, tanto em português como em inglês, unidos por "e" / "and" ou "ou" / "or", a fim de proporcionar uma busca mais amplificada.

Em seguida, procurou-se selecionar os artigos que atenderam aos seguintes critérios de inclusão: (a) a amostra deveria ter como população crianças e adolescentes com deficiência; (b) utilização de um ou mais métodos de mensuração de atividade física; (c) artigos originais de pesquisa com seres humanos; (e) publicação até ano de 2010. Optou-se por não incluir teses, dissertações e monografias, vista a dificuldade de busca sistemática das mesmas.

Os estudos que atenderam plenamente aos critérios de inclusão foram selecionados e posteriormente foram analisados os seguintes temas: (a) ano de publicação do estudo, (b) faixa etária da amostra, (c) tipo de deficiência dos participantes do estudo, (d) delineamento do estudo (e) tipo de método utilizado para mensuração.

Todo o processo de busca, identificação e análise dos dados foram realizadas pelas duas autoras do trabalho. Caso houvesse itens em que não existisse algum consenso, um terceiro pesquisador seria convidado a fazer a possível análise final, o que não foi necessário nesta ocasião.

\section{RESULTADOS}

Dos 70 artigos encontrados e analisados inicialmente, apenas 11 atenderam aos critérios de inclusão neste estudo. Dos estudos que foram excluídos, 13 foram em decorrência da faixa etária da população ser adulta, 25 tratavam de inclusão e barreiras arquitetônicas para a prática de atividades físicas, oito abordavam os benefícios psicológicos da prática de atividade física, quatro retratavam aspectos éticos, filosóficos e políticos e outros nove abordavam o esporte rendimento para pessoas com deficiência.

Dos estudos incluídos na revisão sistemática, todos foram publicados a partir do ano de 2005. A idade da população estudada era em média cerca de 10 anos, sendo que os sujeitos mais novos apresentavam em torno de seis anos e os mais velhos vinte anos de idade.

O tipo de deficiência mais estudado em relação à prática habitual de atividade física foi à deficiência intelectual, seguida da deficiência motora. Todos os estudos tiveram como característica o fato de serem do tipo transversal. Os métodos diretos foram os mais utilizados para verificar os níveis de atividade física, sendo que a primeira opção foi a acelerometria, seguida da pedometria.

A tabela 1 apresenta as características dos estudos incluídos nesta revisão. São destacadas informações referentes ao ano de publicação, tipo de deficiência da amostra, tamanho amostral e método de mensuração utilizado. 


\begin{tabular}{lccccc}
\hline \multicolumn{1}{c}{ Estudo } & Ano & $\begin{array}{c}\text { Faixa Etária } \\
\text { da População }\end{array}$ & Tipo de Deficiência & N & Instrumento \\
\hline Beets et al $^{7}$ & 2007 & 9 a 12 & Intelectual e/ou Motora & 18 & Pedômetro \\
Pitetti, Beets e Flaming $^{8}$ & 2009 & 10 a 16 & Intelectual & 24 & Pedômetro \\
Sit et al. $^{9}$ & 2008 & 10 a 12 & Intelectual & 80 & SOFIT (Observação) \\
Foley e McCubbin $^{10}$ & 2009 & 7 a 12 & Intelectual & 9 & Acelerômetro \\
Kim e Yun $^{11}$ & 2009 & 12 a 20 & Intelectual & 16 & Acelerômetro e Pedômetro \\
Shields, Dodd e Abblitt $^{12}$ & 2009 & 8 a 14 & Intelectual & 23 & Acelerômetro \\
Beets et al $^{13}$ & 2007 & 11 a 15 & Visual & 35 & Pedômetro de voz \\
Van Eck et al. $^{14}$ & 2008 & 12 a 16 & Motora (PC) & 72 & Questionário (Pais) \\
Capio, Sit e Abernethy $^{15}$ & 2010 & 6 a 14 & Motora (PC) & 31 & Acelerômetro \\
Kozub, Oh e Rider $^{16}$ & 2005 & 6 a 18 & Visual & 19 & Acelerômetro \\
Pan $^{17}$ & 2008 & 7 a 12 & Autismo & 53 & Acelerômetro \\
\hline Pr $^{17}$ Parano & & &
\end{tabular}

$(\mathrm{PC}=$ Paralisia Cerebral $)$

\section{DISCUSSÃO}

O principal objetivo desta revisão sistemática foi trazer informações relevantes sobre os diferentes métodos de mensuração de atividade física utilizados em jovens com deficiência. Dentre estes achados, pode-se destacar: (a) o período de realização dos estudos, sendo que todos os encontrados foram realizados nos últimos cinco anos, talvez pelo fato do grau de importância que a atividade física adaptada vem ganhando nos dias atuais; (b) os estudos apresentaram diferentes tipos de métodos de mensuração utilizados pelos autores, sendo o mais utilizado a acelerometria; (c) notou-se que a preferência por parte dos pesquisadores se deu para realização dos estudos em ambiente escolar, o que pode ter ocorrido pela facilidade de se encontrar a amostra na faixa etária pretendida; (d) os estudos tiveram uma duração média de uma a duas semanas; (f) o tipo de deficiência mais investigado foi intelectual.

A seguir alguns apontamentos sobre os métodos de mensuração da atividade física analisados ao longo da revisão. Vale ressaltar que, em todos os estudos levantados, os autores afirmam que os níveis de atividade física apresentados pelos jovens, independente da deficiência, estavam abaixo do ideal recomendado para uma vida saudável.

\section{Observação Direta}

O método de observação direta, de acordo com Guedes e Guedes ${ }^{18}$, consiste em registrar informações associadas à prática da atividade física simultaneamente à ocorrência do evento, por tempo limitado e em ambiente físico claramente definido.

Os procedimentos relacionados à observação direta são mais comumente empregados em crianças na idade pré-escolar ou em sujeitos que não apresentam condição de serem submetidos a outros instrumentos de medida. $O$ estudo encontrado nesta revisão foi o de Sit et al. ${ }^{9}$, onde tal fato não foi diferente. Os pesquisadores se utilizaram do SOFIT (System for Observing Fitness Instruction Time) como método aplicado a crianças e adolescentes para avaliar a qualidade das aulas de educação física e a oportunidade dos alunos serem mais aptos fisicamente. No estudo citado, o instrumento foi aplicado em 80 crianças com deficiência intelectual. Através deste, o observador se utilizou de um sistema de pontuação pautado em três itens que envolveram o tempo em que o indivíduo permaneceu ativo, o contexto da aula de educação física e o comportamento do professor na aula. A observação das crianças se deu no horário das aulas de educação física e no recesso das aulas.

Os resultados da observação apontaram que os alunos mais envolvidos na atividade física apresentaram maiores níveis de intensidade de atividade. As diferenças na prática de atividade física durante a educação física, entre as duas escolas observadas, estavam associadas tanto ao contexto de aula como ao comportamento apresentado pelo professor durante a aula.

Dentre as vantagens apresentadas por este método pode-se citar a oportunidade que o mesmo dá ao observador de registrar a ocorrência dos eventos, o que pode ser feito com menor intervenção do avaliado no registro das informações. Por outro lado, as limitações associadas a este método estão principalmente associadas à qualidade das informações relatas pelos observadores.

\section{Questionário e Entrevista}

Este tipo de instrumento de medida, de acordo com a literatura ${ }^{19}$, possui grande praticidade, sobretudo em situações em que o número de avaliados a serem analisados atinge grandes proporções. Ainda como vantagens, podem-se citar o baixo custo financeiro e a facilidade de se realizar a coleta e analisar os dados. No entanto, as limitações existem, assim como nos outros tipos de métodos. Dentre estas se podem destacar a fidedignidade das informações passadas pelos entrevistados, devido à dificuldade de alguns avaliados se recordarem das atividades realizadas e também à tendência dos mesmos superestimarem suas informações. Em populações jovens tais limitações são ainda mais comuns.

Nesta revisão sistemática, nenhum estudo encontrado fez uso de questionários ou entrevistas aplicadas diretamente aos jovens com deficiência. No estudo de Van Eck et al. ${ }^{14}$, foram aplicados questionários recordatórios de atividades realizadas voltados a mensurar os níveis de atividade física de jovens com paralisia cerebral. Os instrumentos foram respondidos pelos pais destes adolescentes que possuíam deficiência motora. Os autores argumentaram que tal método foi utilizado justamente devido à dificuldade dos adolescentes em observar e também se lembrar das atividades realizadas durante o dia. De acordo com as respostas dos pais, $89 \%$ dos adolescentes não eram fisicamente ativos, principalmente as mulheres e os adolescentes mais velhos. Outro achado do 
estudo foi que a gravidade da paralisia cerebral não estava associada com o desenvolvimento motor e com o nível de atividade dos adolescentes. Os autores do estudo alegaram que isto pode ter ocorrido pelo fato dos familiares incentivarem constantemente estes jovens à prática de atividades físicas.

\section{Sensores de movimentos}

Sensores de movimentos são instrumentos de medida que permitem monitorar a realização de movimentos e oferecem a possibilidade de informações quanto ao dispêndio energético associado. Os sensores de movimentos mais frequentemente empregados como indicadores dos níveis de prática da atividade física são os acelerômetros portáteis e os pedômetros ${ }^{18}$.

As pesquisas realizadas com estes métodos são cada vez mais convincentes devido à sua fidedignidade, pois suas informações são obtidas quanto à quantidade de movimentos realizados pelo indivíduo. Logo, acredita-se que a quantidade de movimentos verificados provavelmente estará mais próxima dos verdadeiros valores de atividade física do avaliado. Tal tendência pode ser observada nesta revisão, uma vez que foi verificado que na maioria dos estudos encontrados os autores se utilizaram de acelerômetros seguidos de pedômetros para mensurar os níveis de atividade física da população estudada.

A avaliação da atividade física com o emprego de acele-

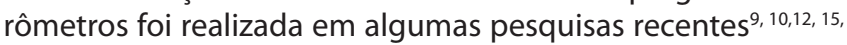
16,17 . Nestes estudos pode - se verificar que este tipo de mensuração foi realizado em um período de uma a duas semanas, afim de que se obtivessem dados dos finais de semana, período este no qual os jovens mostraram ser menos fisicamente ativos. Pode - ser notar ainda que este método foi mais utilizado em adolescentes com deficiência intelectual, seguido dos que tinham deficiência motora. Todavia, foram empregados diferentes tipos de acelerômetros, tanto os uniaxiais como os triaxiais, de diferentes tipos e marcas, o que dificulta a comparação dos níveis de atividade física entre as crianças e adolescentes com algum tipo de deficiência. Em alguns estudos os autores apenas relataram que utilizaram acelerômetros, no entanto, não especificaram se estes eram biaxiais ou triaxiais, nem tampouco suas marcas.

Nos estudos realizados com o método da pedometria ${ }^{7}$, 8, 13 encontraram-se dois tipos de deficiência estudada: a intelectual e a visual. Os resultados mostraram que a escolha do método utilizado para mensuração da atividade física foi positiva de acordo com os autores, uma vez que os dados obtidos foram considerados confiáveis, mesmo estes variando de acordo com os dias da semana, e também com o tipo de deficiência apresentado. No geral os níveis eram maiores nos dias da semana, nos quais os sujeitos tinham aulas de educação física e menores aos finais de semana.

No estudo realizado por Beets et al. ${ }^{13}$, os pesquisadores avaliaram a eficácia de três tipos de pedômetros de voz desenvolvidos por eles em adolescentes com deficiência visual, a fim de que estes pudessem ter uma resposta mais real dos seus resultados relacionados à sua frequência de passos. A precisão dos resultados obtidos com a população estudada foi considerada muito positiva pelos pesquisadores do estudo.

Dentre os estudos realizados com sensores de movimento, somente o de Kim e Yun ${ }^{11}$ adotou os métodos de acelerometria e pedometria ao mesmo tempo com adolescentes com deficiência intelectual. No entanto, devido a erros na metodologia do estudo, tais como uso incorreto dos aparelhos e perda muito elevada de amostra, os resultados não puderam ser comparados entre os métodos. De acordo com os autores, nenhum estudo até o momento examinou a variabilidade intra-individual e inter-confiabilidade de instrumentos de atividade física diária simultaneamente em crianças e adolescentes com deficiência.

Embora os benefícios da prática habitual de atividade física sejam amplamente divulgados na literatura atualmente, os níveis de atividade física apresentados nas pesquisas verificadas se mostraram insatisfatórios. Estudos revelam que jovens com deficiência têm menos oportunidade de realizar atividades físicas e estão mais propensos a apresentar sobrepeso e obesidade. Este fato é preocupante, pois é essencial que em idade escolar jovens com e sem deficiência realizem atividades físicas diárias para a promoção da saúde, além de garantir o crescimento e desenvolvimento motor a fim de se reduzir os fatores de risco para a idade adulta de doenças cardiovasculares e metabólicas ${ }^{20,21}$.

Dada a importância da atividade física, é relevante notar os diferentes métodos de mensuração que foram empregados para a análise da mesma. Evidenciaram-se nos estudos diferentes tipos de métodos utilizados, no entanto a monitorização mais frequente foi a acelerometria, sendo que sua praticidade de aplicação em relação à população em questão e a vantagem deste método apresentar medida direta podem justificar tal escolha, além do fato de os dados não dependerem da recordação dos indivíduos. A confiabilidade do método da acelerometria foi evidenciada no estudo de Kozub, Oh e Rider ${ }^{16}$, no qual os autores verificaram sua validade e confiabilidade em pessoas com deficiência visual e obtiveram respostas positivas em relação aos resultados. No estudo de Foley e McCubbin ${ }^{10}$, a acelerometria também se mostrou um método viável de aplicação para adolescentes com deficiência intelectual. Em todos os estudos, os autores procuraram se utilizar de métodos validados para fazerem a mensuração, dando mais confiabilidade e veracidade aos resultados alcançados.

No estudo de Beets et al. ${ }^{13}$ pode-se verificar a validação do método de pedometria de voz. Este recurso fornece um anúncio de voz audível sobre o número de etapas registradas pelo pedômetro. Estes equipamentos podem ser ajustados para anunciar medidas de acordo com os intervalos pré-definidos (por exemplo, a cada mil passos) ou podem ser acionados através de um botão. Tais características podem ser úteis em determinadas populações para as quais a leitura da exposição não é viável, como a deste estudo, que foram adolescentes com deficiencia visual. Os resultados revelaram que o uso destes pedometros de voz é reprodutível para estudos com este tipo de população.

No entanto, é válido destacar que alguns cuidados com estes métodos devem ser tomados. Por exemplo, indivíduos que fazem uso de cadeira de rodas não poderiam se utilizar dos pedômetros, já que os mesmos contam os números de passadas para calcular os níveis de atividade física. Outro ponto importante é que o pesquisador, além de instruir corretamente os participantes do estudo, deve se atentar ao posicionamento tanto dos pedômetros quanto dos acelerômetros, pois dependendo da deficiência motora apresentada os equipamentos podem não ser muito sensíveis aos movimentos. Em jovens com deficiência intelectual também podem existir dificuldades quanto ao uso de tais equipamentos, especialmente nos casos mais severos.

As metodologias empregadas variaram entre os estudos, mas percebeu-se que o ambiente escolar ainda é o preferido pelos pesquisadores nos trabalhos analisados. Notou-se ainda 
a opção unânime pela realização de estudos transversais, revelando a falta de estudos longitudinais na área de atividade física para pessoas com deficiência.

Embora não tenham sido encontrados estudos que se utilizaram de questionários recordatórios para mensurar a prática habitual de atividade física com crianças e adolescentes com deficiência, é válido destacar que existem instrumentos validados para adultos com deficiência e que estes vêm sendo utilizados por alguns autores 22, 23, 24 .

É interessante perceber que de todos os artigos pesquisados, desde aqueles que foram excluídos da revisão até os selecionados, nenhum deles era de origem brasileira. Este fato reporta assim à realidade da literatura nacional na área de atividade física adaptada, mostrando que esta ainda carece de olhares dos profissionais e dos estudiosos.

A identificação das lacunas existentes serve de alerta para pesquisadores na área da atividade física e saúde, uma vez que as informações obtidas poderiam ser utilizadas para a adoção de medidas de promoção e políticas de saúde, principalmente para a população em questão. Assim, faz-se necessária a realização de estudos futuros para fornecer subsídios e ainda mais resultados sobre os níveis de atividade física de crianças e adolescentes com deficiência.

O presente estudo apresentou como limitação a dificuldade em comparar os resultados apresentados nas pesquisas levantadas, uma vez que foram os métodos empregados, bem como características da amostra, foram muito diferenciados. No entanto, são identificados alguns pontos em comum nos resultados obtidos, tais como o baixo nível de atividade física em geral observado entre jovens com deficiência, o que deve ser motivo de preocupação para os profissionais na área da saúde. Além disso, é possível se observar que, dependendo da deficiência da população a ser analisada, são recomendáveis sistemas diferenciados de mensuração, de forma a obter dados mais precisos e confiáveis. Assim, cabe ao avaliador, tendo em vista as características dos sujeitos do estudo, optar pela utilização de questionários, fichas de observação ou sensores de movimento, de modo que sejam respeitadas as especificidades de cada deficiência.

Por conta das pesquisas expostas, conclui-se que o método da acelerometria tem sido o meio de mensuração mais utilizado pelos pesquisadores para se verificar os níveis de atividade física de jovens com deficiência. Também se notou que a deficiência mais estudada foi a intelectual. Todavia, não existe um consenso na literatura para o tipo de método mais eficaz a ser utilizado, sabendo-se apenas que a decisão depende do tipo de deficiência apresentada pela população e o objetivo do estudo. Sugere-se a realização de mais estudos relacionados a este tema, com diferentes tipos de métodos e deficiências a fim de que seja possível evidenciar os níveis de atividade física apresentados pelas crianças e adolescentes com deficiência.

\section{REFERÊNCIAS}

1. Alves JGB, Montenegro FMUM, Oliveira FA, Alves RV. Práticas de esportes durante a adolescência e atividade física de lazer na vida adulta. Rev Bras Med Esporte 2005; 11 (5): 291-94.

2. Baruki BSB, Rosado LEFPL, Rosado GP, Ribeiro RCL. Associação entre estado nutricional e atividade física em escolares da rede municipal de ensino em Corumbá - MS. Rev Bras Med Esporte 2006; 12 (2): 90-94.

3. Bracco MM, Carvalho KMB, Bottoni, A et al. Atividade física na infância e adolescência: impacto na saúde pública. Rev Ciênc Méd 2003; 12(1): 89-97.

4. Interdonato GC, Greguol M. Auto - Análise da imagem corporal de adolescentes com deficiência visual sedentários e fisicamente ativos. Conexões 2009; 7(3): 1-13.

5. Ginis KAM, Latimer AE, Buchholz AC et al. Establishing evidence-based physical activity guidelines: methods for the Study of Health and Activity in People with Spinal Cord Injury (SHAPE SCI). Spinal Cord 2008; 46 (1): 216-21.

6. Cervantes $\mathrm{CM}$, Porretta DL. Physical activity measurement among individuals with disabilities: a literature review. Adapt Phys Activ Q 2010; 27 (4): 173-90.

7. Beets MW, Combs C, Pitetti KH et al. Accuracy of pedometer steps and time for youth with disabilities. Adapt Phys Activ Q 2007; 24 (3): 228-44.

8. Pitetti $\mathrm{KH}$, Beets MW, Flaming J. Accuracy of pedometer steps and time for youth with intellectual disabilities during dynamic movements. Adapt Phys Activ Q 2009; 26 (4): 336-51.

9. Sit $\mathrm{CH}$, McKenzie TL, Lian JM, McManus A. Activity levels during physical education and recess in two special schools for children with mild intellectual disabilities. Adapt Phys Activ Q 2008; 25(3): 247-59.

10. Foley JT, McCubbin JA. An exploratory study of after-school sedentary behaviour in elementary school-age children with intellectual. J Intellect Dev Disabil 2009; 34(1): 3-9.

11. Kim SY, Yun J. Determining daily physical activity levels of youth with developmental disabilities: days of monitoring required? Adapt Phys Activ Q 2009; 26(3): 220-35

12. Shields N, Dodd KJ, Abblitt C. Do children with down syndrome perform sufficient physical activity to maintain good health? A pilot study. Adapt Phys Activ Q 2009; 26(4): 307-20

13. Beets MW, Foley JT, Tindall DWS, Lieberman LJ. Accuracy of voice - announcement pedometers for youth with visual impairment. Adapt Phys Activ Q 2007; 24(3): 218-27.

14. Van Eck M, Dallmeijer AJ, Beckerman $\mathrm{H}$ et al. Physical activity level and related factors in adolescents with cerebral palsy. Pediatr Exerc Sci 2008; 20(1):95-106.

15. Capio CM, Sit CH, Abernethy B. Physical activity measurement using MTI (actigraph) among children with cerebral palsy. Arch Phys Med Rehabil 2010; 91(8): 1283-90.

16. Kozub FM, Oh HK, Rider RA. RT3 accelerometer accuracy in estimating shor term physical activity in individuals with visual impairments. Adapt Phys Activ Q 2005; 22(3): 265-76.

17. Pan CY. School time physical activity of students with and without autism spectrum disorders during PE and recess. Adapt Phys Activ Q 2008; 25(4): 308-21.

18. Guedes DP, Guedes JERP. Manual prático para avaliação em educação física. Barueri, SP: Manole, 2006.

19. Tassitano RM, Bezerra J, Tenório MCM et al. Atividade física em adolescentes brasileiros: uma revisão sistemática. Rev Bras Cineantropom Desempenho Hum 2007; 9(1): 55-60.

20. Rimmer J, Riley BB, Rubin SS. A new measure for assessing the physical activity behaviors for persons with disabilities and chronic health conditions: the physical activity and disability survey. Am J Health Promot 2001; 16 (1): 34-42.

21. Rimmer J, Rowland J, Yamaki, K. Obesity and Secondary Conditions in Adolescents with Disabilities: Addressing the Needs of an Underserved Population. Int J Child Adolesc Health 2007; 41(1): 224-29.

22. Washburn RA, Zhu W, McAuley E et al. The physical activity scale for individuals with physical disabilities: development and evaluation. Arch Phys Med Rehabil 2002; 83(2): $193-200$

23. Stevens SL, Caputo JL, Fuller DK, Morgan DW. Physical Activity and Quality of Life in Adults with Spinal Cord Injury. J Spinal Disord Tech 2008; 31(4): 373 - 78.

24. Van der Ploeg HP, Streppel KR, Van der Beek et al. The physical activity scale for individuals with physical disabilities: test-retest reliability and comparison with an accelerometer. J Phys Act Health 2007; 4(1): 96 - 100 